\title{
Apps to Teach Social Skills to Individuals with Autism Spectrum Disorder: A Review of the Embedded Behaviour Change Procedures
}

\author{
Nicole Hanna ${ }^{1} \cdot$ Helena Lydon ${ }^{1} \cdot$ Jennifer Holloway ${ }^{1,2}\left(\mathbb{D} \cdot\right.$ Lorna Barry $^{3} \cdot$ Edith Walsh $^{4}$
}

Received: 18 October 2020 / Accepted: 20 May 2021 / Published online: 5 June 2021

(c) The Author(s) 2021

\begin{abstract}
Social skill interventions have become increasingly popular in recent times, as well as the use of technology to deliver and aid interventions for the autism spectrum disorder (ASD) population. Little research exists on the use of apps to teach social skills to individuals with ASD, in particular the behaviour change procedures that exist within apps. The current review examines the behaviour change procedures that exist within apps according to the Behavior Analyst Certification Board Task List, 5th Edition. A total of 15 apps were included within this review. Results indicate that a variety of behaviour change procedures exist within apps. However, the quality of apps and the number of behaviour change procedures vary across app developers.
\end{abstract}

Keywords Social skill interventions · Behaviour change procedures · Apps · Task List 5th Edition

Autism spectrum disorder (ASD) is a developmental disorder that is characterized by deficits in social communication and social interaction, and restricted, repetitive patterns of behaviour, interests, or activities (McCoy et al., 2016). The Diagnostic and Statistical Manual of Mental Disorders, 5th Edition (DSM-5) outlines that individuals who present with ASD often have deficits in social-emotion reciprocity; deficits in nonverbal communicative behaviours; and deficits in developing, maintaining, and understanding relationships (American Psychiatric Association, 2013). Individuals with ASD frequently have difficulty initiating and responding to social interactions, sustaining eye contact, responding to other's feelings, comprehending, and responding to nonverbal communication and deficits in social problem solving (Boyd et al., 2008; Camargo et al., 2014). ASD can vary in severity and different deficits exist across a broad spectrum; therefore, the research using interventions that target social skills varies considerably depending on the individual's current

Jennifer Holloway

jennifer.holloway@nuigalway.ie

1 Applied Behavior Research Clinic, National University of Ireland Galway, Galway, Ireland

2 School of Psychology, National University of Ireland Galway, Galway, Ireland

3 i-TEACH Lab, University of Limerick, Limerick, Ireland

4 Athlone Institute of Technology, Athlone, Ireland capabilities and skill set. Crooke et al. (2008) highlighted that interventions differ from skill-based approaches for teaching social skills for individuals with limited or emerging language to interventions on more social cognitive tasks for those with more complex language abilities. Social skills deficits do not remit across an individual's life span in the absence of intervention; instead, these deficits can cause other impairments and distress as the individual develops and becomes more aware of these deficits (Williams White et al., 2007). Long-term and without direct intervention, these social deficits can result in problems in reciprocal peer relationships, a difficulty to demonstrate empathy and weak integration of social, emotional, and communicative behaviours (Ramdoss et al., 2012).

Current literature reviews suggest that no one social skill intervention is suitable for all individuals with ASD, and currently, there is no common, agreed upon approach to teach social skills to individuals with ASD (Flynn \& Healy, 2012). The efficacy of a variety of interventions to increase social skills of individuals have been examined including, peer mediated interventions (PMI), social skills groups (SSG), pivotal response training (PRT), script fading, video modelling (VM) and reinforcement-based procedures (Flynn \& Healy, 2012), structured teaching, and developmental/ relationship interventions (Walton \& Ingersoll, 2013). Reichow and Volkmar's (2010) evaluation of evidencebased practice within social skills interventions highlighted that the most frequently used intervention techniques were 
based on Applied Behavior Analysis (ABA) principles such as prompting and reinforcement. The authors further highlighted that $\mathrm{ABA}$ principles were also used to augment other intervention types and therefore should be continued to be used in social skill interventions (Reichow \& Volkmar, 2010). Treatments for individuals with ASD can be intensive and costly for parents and caregivers with limited resources available free of charge (Hourcade et al., 2013); therefore, it is vital to have a tool to refer to when examining interventions and examining the cost benefit of interventions. The Behavior Analyst Certification Board (BACB) has devised a Task List, 5th Edition (TL5) that is divided into two major sections. Section 1 Foundations includes (a) philosophical underpinnings; (b) concepts and principles; (c) measurement, data display, and interpretation; and (d) experimental design; and Sect. 2 Applications includes (e) ethics, (f) behaviour assessment, (g) behaviour change procedures, (h) selecting and implementing interventions, and (i) personnel supervision and management (Behavior Analyst Certification Board, 2017). The task list is developed based on job analyses, where individuals within the field are selected based on their experience and expertise across the field of behaviour analysis. These individuals are brought together to identify the key functions and duties within the field of behaviour analysis at that particular time. These professionals represent the latest practices or trends that are common among practitioners, while also highlighting content that is not widely accepted within the field and excludes procedures and practices that are not yet evaluated through research (Johnston et al., 2014). Section G lists each behaviour change procedure widely used among clinicians and practitioners within the field and includes mainly practice-oriented skills. Recently, innovative advancements in technology have paved the way for more cost-effective interventions for individuals with ASD as well as providing accessible interventions that target various outcomes and specific deficits (Wainer \& Ingersoll, 2011). This advancement, along with the observation that individuals with ASD show an affinity to technology, led researchers to recognize computers as an effective and efficient tool in treatment (Ploog et al., 2013). Ploog et al. (2013) review of computer-assisted technologies (CATs) found that the most common technologies that are employed to teach individuals with ASD social skills are video modelling and virtual reality. Video modelling has extensive empirical evidence for increasing social skills in individuals with ASD, in particular with children and adolescents, showing increases in answering and asking questions, giving compliments, social initiation, and reciprocity (Mason et al., 2012).

In the past decade, there have been a number of reviews examining the use of technology and computer-assisted interventions to teach social skills to individuals with ASD (Grossard et al., 2017; McCoy et al., 2016; Ramdoss et al.,
2012; Reed et al., 2011). Reed et al. (2011) review of using technology to teach social skills categorized social skills into the topography of each social skill targeted, resulting in 9 categories: (1) initiating conversations; (2) responding to others initiations through conversations; (3) social conventions as part of conversation (staying on topic); (4) nonverbal behaviours; (5) play skills; (6) social problem solving; (7) friendship/peer relationship (8) emotion identification, regulation, and reciprocity; and,(9) other social skills not outlined above. Results indicated that the most common social skills taught were conversational skills followed by play skills (Reed et al., 2011). Ramdoss et al. (2012) further examined the existing research on the use of computer-based interventions (CBI) to teach social and emotional skills to individuals with ASD. They found that using CBI to teach social skills showed positive results; however, concerns were highlighted as the literature failed to examine the generalization of these skills to real-life scenarios (Ramdoss et al., 2012). A more recent review and in-depth evaluation of video modelling, role play, and $\mathrm{CBI}$ interventions to teach social skills to individuals with high-functioning autism (HFA) found each intervention to be successful in targeting a variety of social skills, such as increasing sportsmanship and conversational turn taking (role play); recognition of body language and facial expression in others (VR); and perspective taking, greetings, and social initiations (VM) (McCoy et al., 2016). McCoy and colleagues further highlighted that although these interventions showed positive outcomes, CBI was the only intervention to meet Reichow's (2011) criteria for evidence-based practices. Lastly, a review of games to teach social interactions and emotions to individuals with ASD identified 31 games designed to teach social skills, 16 of which targeted emotion recognition and 15 of which targeted more general social skills such as interaction, collaboration, and adaption to specific social contexts, with the majority of these 15 focusing on collaborative skills such as negotiation, turn taking, and planning together (Grossard et al., 2017).

Advancements in the use of technology for teaching social skills to individuals with ASD have resulted in a growing number of applications targeting this specific repertoire. Shic and Goodwin (2015) noted that the popularity of apps has extended to the ASD population with increasingly more apps being developed and made commercially available for individuals with ASD and their families. The demand for social skills apps has increased due to the recent focus on using technology to teach social skills to those with ASD (Mintz, et al., 2012). Parents and clinician's satisfaction with the use of iPads as an education tool has further opened the market further for app developers (Draper Rodríguez et al., 2014). Hourcade et al. (2013) review of tablet apps to increase social interaction noted that the use of apps to teach social skills are helpful in relieving uncertainty and 
the anxiety that real-life interactions may cause. Participants felt that interacting with the tablet was easier, more predictable, and controllable than interacting with people (Hourcade et al., 2013). Although more apps are being developed that target social skills, it is important to ensure these apps are of high-quality and incorporate evidence-based practices that target behaviour change. Hourcade et al. (2013) noted that although the apps had positive effects, this does not mean that tablet apps will benefit children with ASD. Interventions and treatments are increasingly being held to high standards such as "evidence-based practices", and the goal of research should be to provide interventions that are as effective as possible for clients and their families (Mesibov \& Shea, 2011). This goal should extend to apps and computer-based interventions. Odom et al. (2015) highlighted that questions currently exist around applications and whether they meet current evidence-based practice standards. Many social skills apps that exist are not specifically designed for individuals with ASD (Ploog et al., 2013). Kim et al. (2018) found that only $4.9 \%$ of 700 apps labelled as evidence-based "Autism Apps" were found to have any actual clinical evidence. However, this does not conclude that these apps are harmful or ineffective; instead, it highlights that practitioners should examine apps and the risks and benefits of using them more closely (Kim et al., 2018) as individuals with ASD have the right to effective treatment and treatments that are scientifically validated (Van Houten et al., 1988). The lack of reviews examining the evidence-based procedures within apps is also a cause for confusion for parents who seek apps as a replacement for direct intervention. Dillenburger et al. (2004) examination of parent's perceptions of ABA interventions highlighted the importance for parents to have a knowledge of basic ABA principles in order to individualize treatment for their children as opposed to a "onesize-fits-all" treatment approach which parents without this knowledge may fall victim to. Papadakis and Kalogiannakis (2017) further noted that many apps that are marketed as educational are often a digital or interactive replica of work sheets and lessons that educators already use. Research has suggested that many apps do not keep their promise of supporting learning and evaluating each app is difficult due to the large number of self-proclaimed educational apps. This may potentially exploit parents who may associate cost with effectiveness (Papadakis \& Kalogiannakis, 2017).

More recently, focus has been directed towards identifying effective and scientific techniques present within apps, specifically the behaviour change techniques and procedures. The Behavior Change Technique Taxonomy (BCTT) was developed to provide a shared, standardized terminology to be used to specify the active ingredients of behaviour change interventions (Scott et al., 2020). The BCTT has been used to determine the behaviour change techniques present within apps for physical activity, with results showing fewer than four techniques present in the descriptions of the apps reviewed (Conroy et al., 2014). Similarly, Morrissey et al. (2016) investigated the behaviour change techniques employed within apps for medication adherence, and they identified that only 12 out of a possible 96 behaviour change techniques were present across apps. At present, the literature is lacking in research on social skills apps and the behaviour change procedures (BCP) present in the available apps. Research has suggested that ABA principles are most commonly used within social skill interventions (Reichow \& Volkmar, 2010) along with further research highlighting the importance of parent's knowledge of ABA and its principles (Dillenburger et al., 2004). Therefore, it is vital for current research to explore the existing BCP within apps that are marketed to teach social skills to individuals with ASD.

The aim of the current review is to examine the apps that exist for both Apple and Android devices that are marketed to teach social skills to individuals with ASD. The current review build on previous research by Hourcade et al. (2013) and Kim et al. (2018) by examining the BCP as outlined by the BACB TL5 employed within social skills apps. Apps will be categorized and reviewed based on the $\mathrm{BCP}$ that are embedded within the apps. The purpose of this review was to analyze the $\mathrm{BCP}$ that are embedded within the existing apps for teaching socials skills to individuals with ASD.

\section{Method}

\section{Search Procedure}

Systematic searches were completed in May 2020 of the Apple "iTunes" store and Google "PlayStore" to examine the existing apps relating to teaching social skills in individuals with autism. Apps included in this review are all currently available for download in July 2020 from each app store. App stores were individually searched using the search words "Social Skills". The description of each app on the app stores was screened and was included if they met the inclusion criteria listed below. Following initial screening, apps were downloaded and further assessed for inclusion criteria and the presence of $\mathrm{BCP}$.

\section{Inclusion and Exclusion Criteria}

Apps were required to meet the following inclusion criteria: (a) the apps were designed to teach social skills (this included conversation skills, e.g. initiating/maintaining conversations, how to finish a conversation; friendship skills, e.g. joining a game, going to a friend's house, being a gracious loser; perspective taking, e.g. navigating others expected and unexpected behaviour, understanding behaviours that others may find uncomfortable; social interactions, 
e.g. personal space, recognizing social cues), (b) were marketed for ASD, and (c) were a follow-on from an original app, e.g. beginner, intermediate or advanced versions of apps; these apps were included and rated separately to the original app. Apps were excluded if they (a) included content unrelated to changing social behaviours in people with ASD; (b) focused solely on teaching emotions as a target skill; (c) taught social expectations in isolation, e.g. social stories; (d) were not a stand-alone app, (e) were unable to use, i.e., apps that were freezing or crashing; (f) were not available in English; (g) were only available to download in a specific country (as this would not allow for generalization); and (h) required membership of a specific curriculum or school to use.

\section{Data Extraction}

Each app selected for inclusion was summarized in terms of (a) age range and price; (b) platform on which it is available; (c) social skill(s) targeted; (d) category of BCP; (e) specific BCP; (f) task list item code; and (g) how the BCP was presented.

The BACB task list is divided into two major sections: Sect. 1 Foundations and Sect. 2 Applications which includes section ( $\mathrm{g}$ ) behaviour change procedures (Behavior Analyst Certification Board, 2017). As the current review focused on skills acquisition of social skills for individuals with ASD, apps that were selected for inclusion within this review were examined and coded according to ( $\mathrm{g}$ ) BCP. The BCP listed in this section of the BACB task list was further categorized for the content analysis of this review (see Table 2). These categories were:

1. Antecedent-based interventions, e.g. motivating operations, instructions, and rules.

2. Procedures to increase skill acquisition, e.g. modelling, shaping, prompts.

3. Reinforcement-based procedures to strengthen behaviours, e.g. positive reinforcement, differential reinforcement of high rate behaviour (DRH).

4. Reinforcement-based procedures to weaken behaviours, e.g. differential reinforcement of low rate behaviour (DRL), differential reinforcement of other behaviour (DRO).

5. Programming for generalization and maintenance, e.g. response generalization, maintenance procedures.

Two raters (a graduate student in Applied Behavior Analysis and a doctoral student in Applied Behavior Analysis who is a board-certified behaviour analyst) performed the data extraction independently in June 2020. BCPs were coded using the BACB TL5; apps were trialled independently by each rater and screened for the presence of BCPs.

\section{Quality Assessment}

To examine the quality of the apps included, the Mobile App Rating Scale (MARS; Stoyanov et al., 2015) was used. The MARS is a standardized assessment tool that has been used to rate the quality of health apps, and is an easy to use and widely applicable measure of app quality. The MARS consists of 7 sections (A-F), assessing the following areas: (a) engagement, (b) functionality, (c) aesthetics, (d) information, (e) subjective quality, and (f) assess the perceived impact on the user's knowledge, attitudes, and intentions to change. Each section contains 3-7 items, and items are scored by the raters on a 5-point scale from 1 "inadequate" to 5 "excellent". Additional descriptors are provided for each item to assist with scoring based on the feature of the app being examined. A mean score for each section (a-f) is calculated, then mean scores for Sections A-D are combined to provide an app quality mean score and Section $\mathrm{E}$ is used to provide an app subjective quality score. Section F is excluded from scoring but provides additional app-specific information.

\section{Reliability of Search Procedures and Inter-Rater Agreement}

Inter-rater agreement (IRA) was conducted by two independent raters. Agreement was defined as both raters identifying the same apps to be included within the review. IRA was performed on three states of the review: inclusion/exclusion criteria, data extraction, and quality assessment.

Firstly, each rater independently searched both platforms and screened the app names and descriptions for inclusion criteria. Each rater then produced a list of apps that should be considered for inclusion. Agreement was calculated by calculating the sum of number of apps considered for inclusion by both raters and dividing this sum by the number of apps that were in agreement that were identified by the raters. IRA for the apps considered for inclusion in the review was calculated at $80 \%$. Any disagreements on apps to be included was resolved by a third rater (a board-certified behavoiur analyst - doctorate level) who examined each app based on the inclusion and exclusion criteria and further calculating agreement at $100 \%$. Each app considered for inclusion was downloaded and used independently by both raters. Agreement was defined as both raters identifying the same apps to be excluded once downloaded and screened. Agreement was calculated by adding the number of apps chosen to be excluded by each rater and dividing it by the number of apps that were in agreement. IRA for the apps to be included within the review was calculated at $100 \%$. 
Secondly, for data extraction, agreement was defined as both raters identifying the same BCP embedded within each app. A random sample of 33.3\% $(n=5)$ of included apps was generated. The second rater extracted the information from each app in the random sample. IRA was then calculated to determine the accuracy of the data extracted. IRA on data extraction was $100 \%$.

Thirdly, IRA was also conducted on the MARS quality assessment on the same random sample used for data extraction. IRA was calculated by adding the number of questions scored on the MARS that were in agreement and dividing it by the total number of questions to be scored. IRA on the MARS was calculated at $61.8 \%$.

\section{Results}

A total of 24 apps were initially identified as consistent with the inclusion criteria for this review. Once apps were downloaded for data extraction, a further 9 apps were excluded, resulting in the inclusion of 15 apps (see Fig. 1). Reasons for exclusion included apps not being a full version $(n=1)$, apps freezing/not loading $(n=1)$, apps providing instructions/tips rather than targeting behaviour change $(n=2)$, apps requiring membership of a curriculum $(n=1)$, apps using social stories $(n=3)$, and apps not targeting social skills $(n=1)$. Of the apps included, 33.3\% $(n=5)$ specified a target age group with ages ranging from 2 to 12 years old (see Table 1). A large portion of apps included did not include a specific age
Fig. 1 Prisma diagram of app inclusion

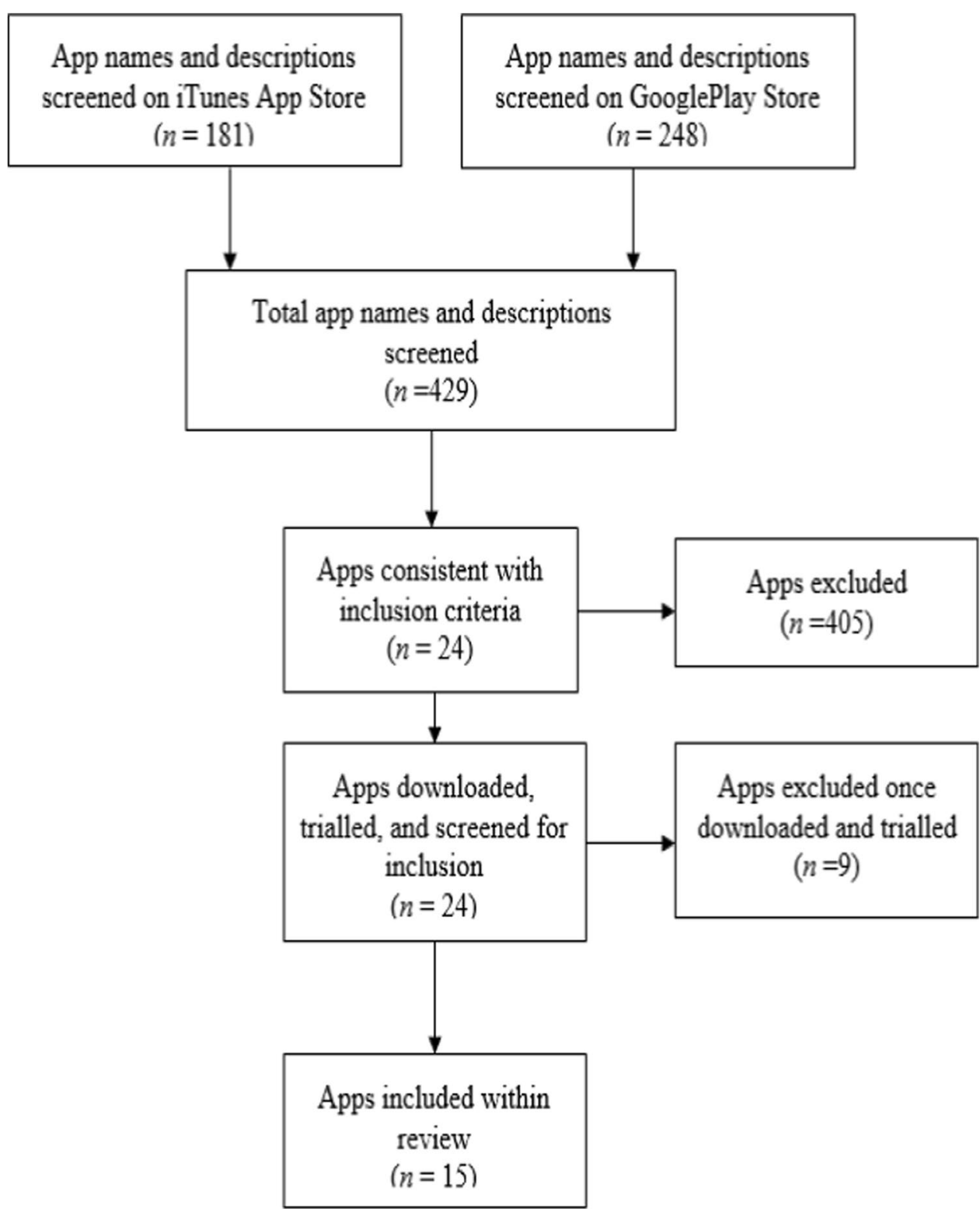




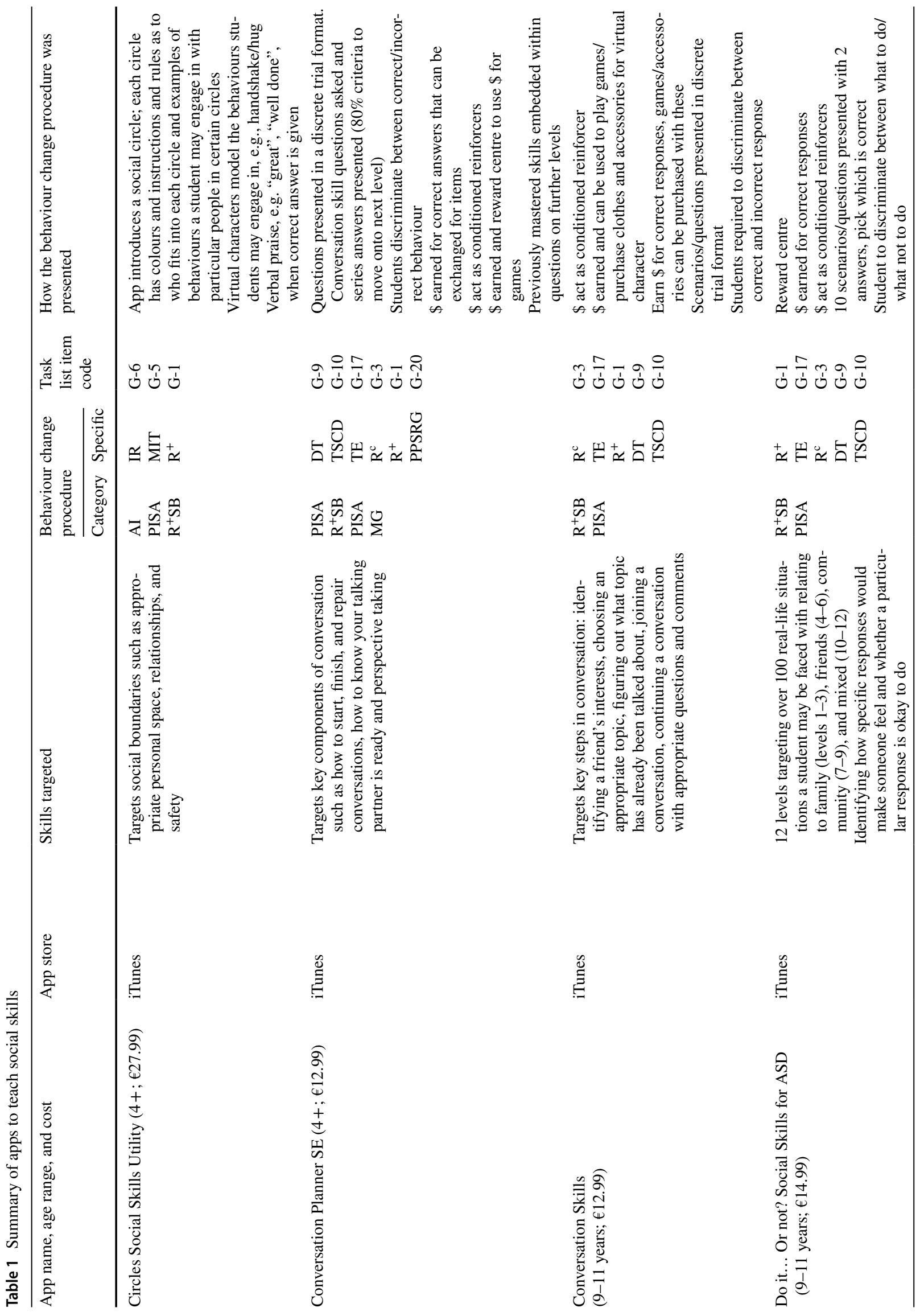




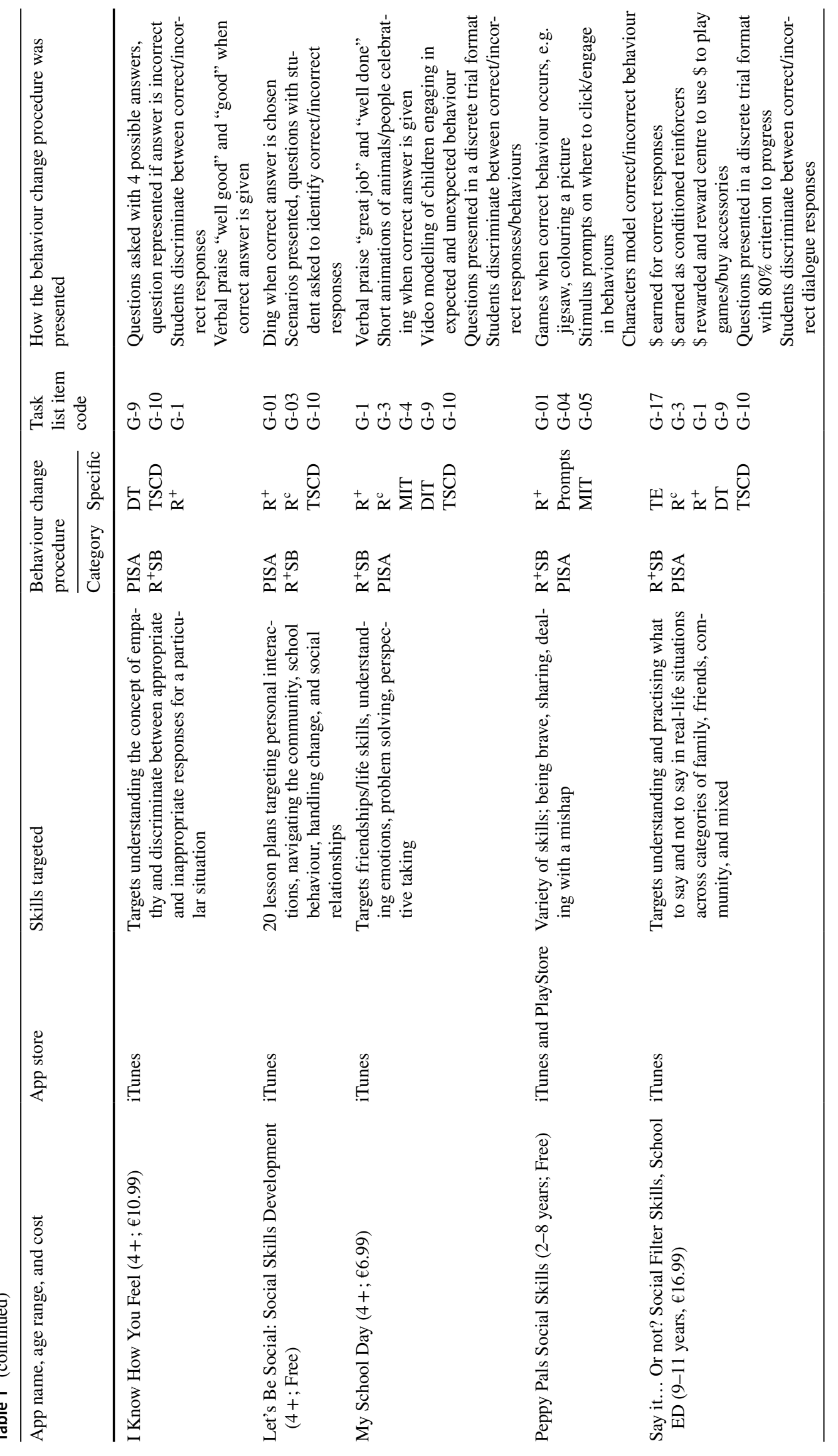




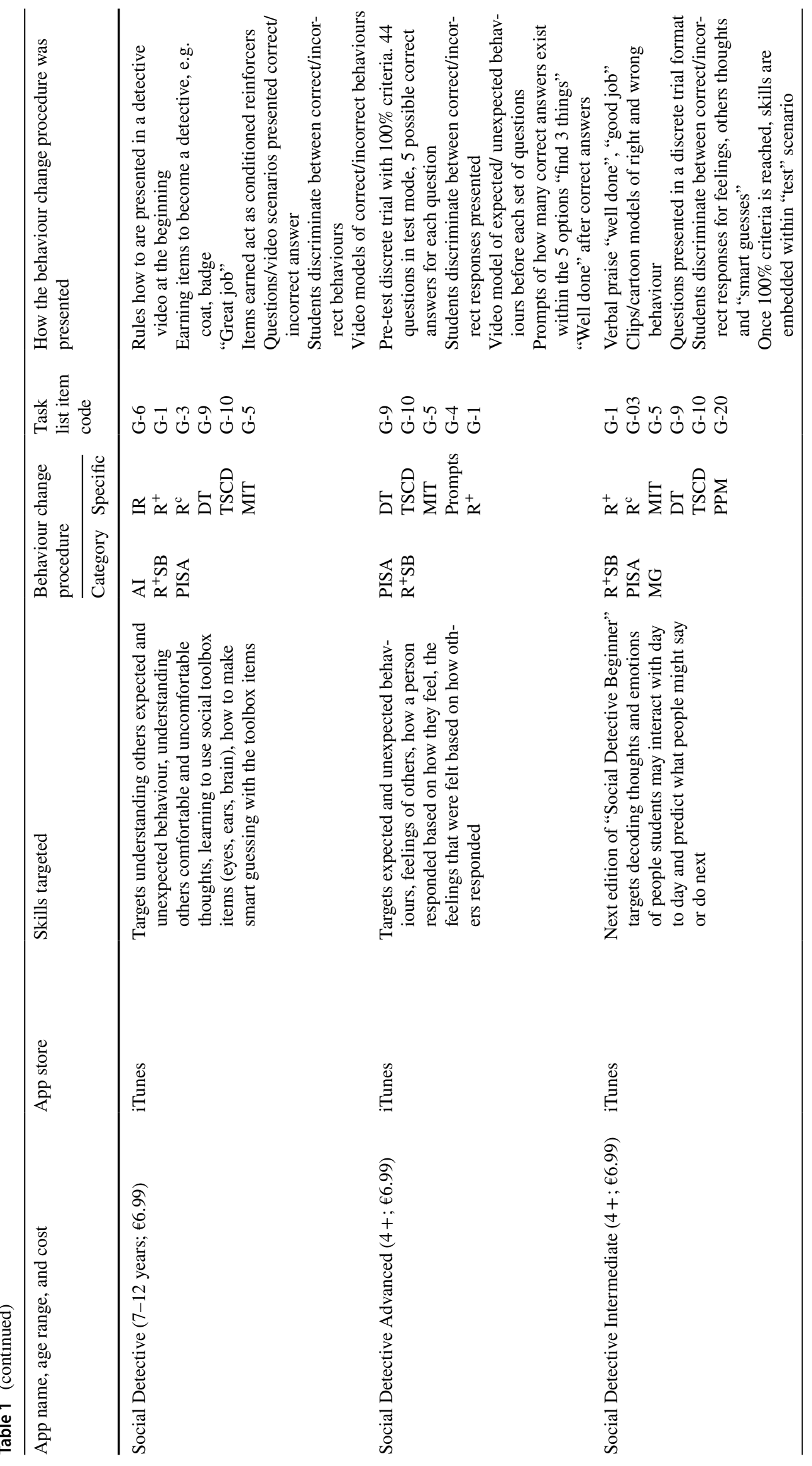




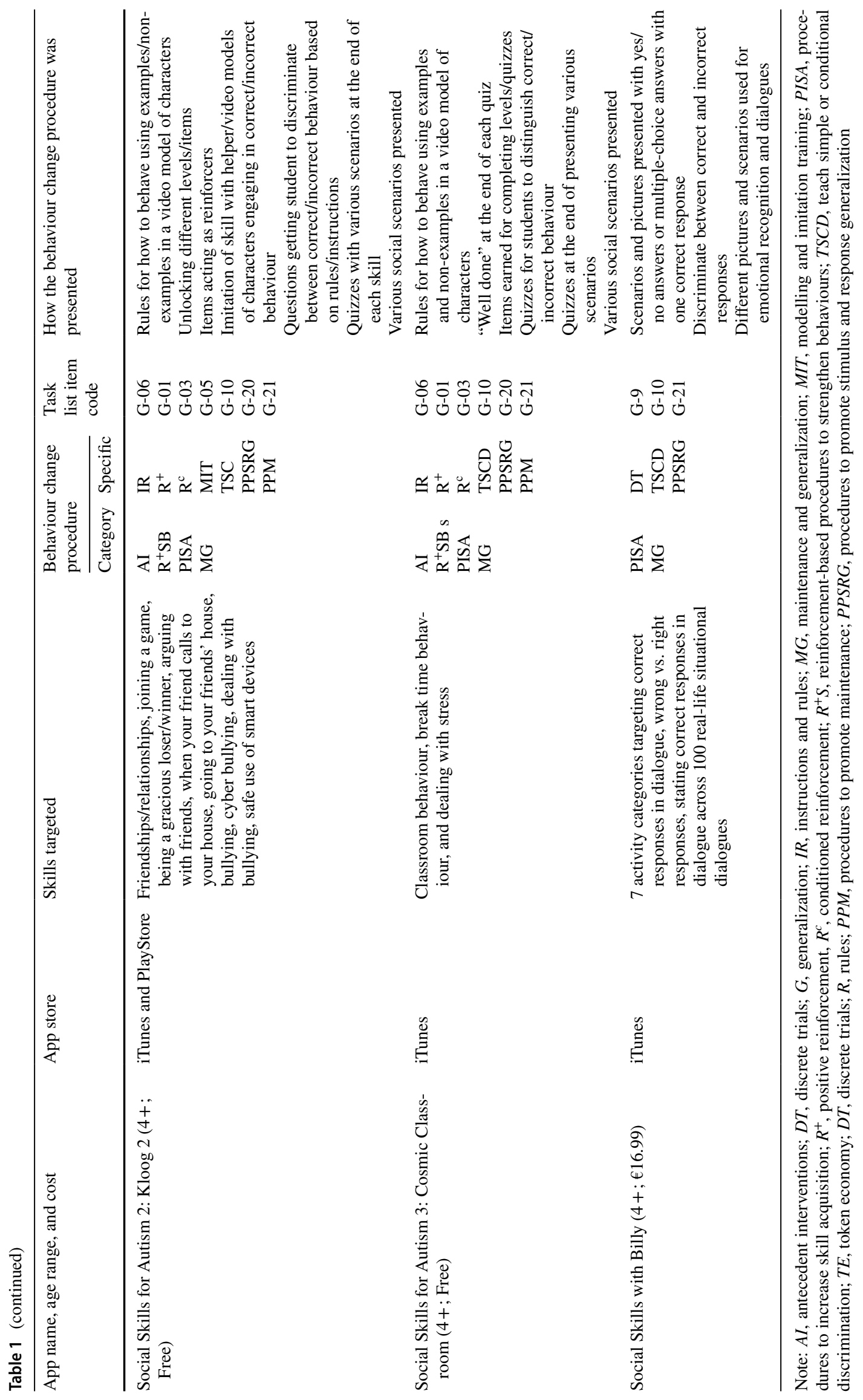


rating within their description with $66.6 \%(n=10)$ subject to the automatic app store rating of ages $4+$. Four apps were free to download (26.6\%), and 11 apps $(73.3 \%)$ required payment with a mean price of $€ 12.80$ and a range of $€ 21.00$ (see Table 1). All apps $(n=15)$ included in this review can be found on the iTunes app store; $20 \%$ of apps $(n=3)$ are available on both the PlayStore and iTunes. No apps were solely available to the PlayStore $(n=0)$ (see Table 1$)$.

\section{Target Skills}

A variety of social skills were found to be targeted within the included apps. The most common skill targeted was understanding the behaviour of others, such as understanding others' expected/unexpected behaviour, feelings of others, and empathy with 5 apps (33.3\%) targeting these skills. Conversation skills such as correct/incorrect responses in dialogue and initiating conversation was targeted in 4 apps (26.6\%). Friendship/relationship skills such as being a gracious loser, sharing, personal space, and social behaviours was targeted in 4 apps $(26.6 \%)$ and social behaviour in school such as following rules and appropriate classroom behaviour was targeted in 2 apps (13.3\%). Although app descriptions described the skills that the app targeted, many did not directly teach the target skill. Instead, apps presented quizzes and questions on various skills without prior direct teaching of the skill, this was evident in $53.3 \%(n=8)$ of apps included; the remaining $46.6 \%(n=7)$ incorporated procedures to demonstrate the skill prior to quizzes and questions.

\section{Categories of Behaviour Change Procedures}

The most common categories of BCP found within apps to teach social skills were procedures to increase skill acquisition with all apps including these procedures ( $n=15 ; 100 \%)$ (see Table 2). This was closely followed by reinforcementbased procedures to strengthen behaviours which were found in $14(93.3 \%)$ apps. Apps also included antecedent interventions in 4 apps (26.6\%) and similarly maintenance and generalization procedures present in 4 apps (26.6\%). Reinforcement-based procedures to weaken behaviours is the only category of $\mathrm{BCP}$ not present in apps to increase social skills.

\section{Specific Behaviour Change Procedures}

The TL5 consists of $22 \mathrm{BCP}$; Table 2 presents a summary of the BCP and number of apps they are present in. Of the 22 BCP, 45.4\% $(n=10)$ can be found in apps that teach social skills to individuals with ASD with a mean of 4.4 BCP in each app. The most commonly used BCPs included positive reinforcement $(93.3 \% ; n=14)$, the use of simple or conditional discrimination $(86.6 \% ; n=13)$, the use of discrete trials $(66.6 \% ; n=10)$, and the use of modelling and imitation $(46.6 \% ; n=7)$. Other BCPs which presented less in apps included use of token economies $26.6 \%(n=4)$, use of instructions and rules $26.6 \%(n=4)$, use of procedures to promote maintenance $26.6 \%(n=4)$ and prompts $13.3 \%$ $(n=2)$; and the use procedures to promote generalization $6.6 \%(n=3)$ which were also found in the included apps.

\section{How Behaviour Change Procedures Are Presented}

There were 10 specific BCP identified within apps (see Table 2). Results indicate that BCP are presented in similar ways across apps. Discrete trials were presented in the same format across apps by incorporating them as quizzes. A trial was presented where an antecedent is presented (the question); individuals are required to engage in a behaviour (choosing a correct answer for a multiple-choice question) and a consequence is presented (ding when correct/verbal praise/conditioned reinforcer). Similarly, discrimination training was presented in the same way across all apps. Discrimination training was used within quizzes throughout apps where a question was presented, and the user is required to discriminate between multiple correct and incorrect responses for the appropriate social behaviour.

The BCP of modelling was presented the same across all apps, using video models of real-life and cartoon characters to model correct and incorrect social behaviours. The use of rules was incorporated within the video models where the characters explained inappropriate and appropriate social behaviours and their consequences. Rules were introduced through a video model of a virtual character such as an alien or detective where appropriate and inappropriate forms of social behaviours were discussed and consequences of these appropriate and inappropriate social behaviours were discussed. Prompts were incorporated within apps in a variety of ways. Prompts were presented as stimulus prompts where an app used flashes of lights, virtual characters looking a certain direction etc. to show the student where to click and the correct behaviours to engage in. Prompts were also incorporated as response prompts where the user was presented with a multiple-choice question where various correct answers exist, a prompt of "choose 4 answers" or "choose 3 answers" to indicate how many responses were required.

Positive reinforcement was most commonly presented in apps in the form of earning items such "\$" rewards, earning access to games, and unlocking different levels $(n=8)$; positive reinforcement in the form of verbal praise such as "well done" and "great job" was also evident $(n=6)$. Conditioned reinforcers were presented in a similar way as positive reinforcement. Reinforcers such as earning items, tokens, and access to other levels were considered conditioned reinforcers from their repeated use as reinforcers throughout the 
Table 2 Summary of categories of behaviour change and behaviour change procedures

\begin{tabular}{|c|c|c|c|}
\hline Category & Behaviour change procedure & TL5 code & Number of apps \\
\hline \multirow[t]{6}{*}{ Antecedent interventions } & & & 4 \\
\hline & Motivating operations & G-02 & 0 \\
\hline & High probability instructional sequence & G-13 & 0 \\
\hline & Instructions and rules & G-06 & $4(26.6 \%)$ \\
\hline & Functional communication training & G-14 & 0 \\
\hline & Non-contingent reinforcement & G-14 & 0 \\
\hline \multirow[t]{7}{*}{ Procedures to increase skill acquisition } & & & $15^{*}$ \\
\hline & Use of positive and negative reinforcement & G-01 & $14(93.3 \%)$ \\
\hline & Use stimulus and response prompts and fading & G-04 & $2(13.3 \%)$ \\
\hline & Use of modelling and imitation training & G-05 & $7(46.6 \%)$ \\
\hline & Use shaping & G-07 & 0 \\
\hline & Use chaining & G-08 & 0 \\
\hline & Teach simple or conditional discrimination training & G-10 & $13(86.6 \%)$ \\
\hline \multirow[t]{6}{*}{ Reinforcement-based procedures to strengthen behaviours } & & & 14 \\
\hline & Establish and use conditioned reinforcers & G-03 & $9(60 \%)$ \\
\hline & Use token economies & G-17 & $4(26.6 \%)$ \\
\hline & Discrete trial & G-09 & $10(66.6 \%)$ \\
\hline & DRA & G-14 & 0 \\
\hline & Self-management & G-20 & 0 \\
\hline \multirow[t]{5}{*}{ Reinforcement-based procedures to weaken behaviours } & & & 0 \\
\hline & Use positive and negative punishment & G-16 & 0 \\
\hline & Use extinction & G-15 & 0 \\
\hline & DRO & G-14 & 0 \\
\hline & DRL & G-14 & 0 \\
\hline \multirow[t]{3}{*}{ Maintenance and generalization } & & & 4 \\
\hline & $\begin{array}{l}\text { Use procedures to promote stimulus and response } \\
\text { generalization }\end{array}$ & G-21 & $3(6.6 \%)$ \\
\hline & Use procedures to promote maintenance & G-20 & $4(26.6 \%)$ \\
\hline
\end{tabular}

Note: $D R A$ differential reinforcement of alternative behaviour, $D R O$ differential reinforcement of other behaviour, $D R L$ differential reinforcement of lower rates of behaviour

*More than one BCP was present within each of the 15 included apps

app. Token economies were embedded within the procedure of discrete trials and discrimination training. However, it should be noted that although discrete trials were present in 10 apps and discrimination training was present in 13 apps, token economies were only present in 4 of these apps. Tokens in the form of " $\$$ " coins were earned for correct responses, and these tokens could be exchanged to buy items for the student's virtual character or to play a game. Token economies were consistently presented in this manner across all apps they appeared in.

Maintenance was presented in the same way across all apps. Apps that included maintenance presented it by embedding previously mastered questions or skills within quizzing on various other levels that targeted new, not previously mastered skills. Generalization was presented within apps by incorporating various scenarios, pictures, and questions based on the same skill. For example, various scenarios were presented across family and friend scenarios and users were presented various quizzes and questions on.

\section{App Developers}

Although 15 apps are included in this review, only 6 app developers exist. Janine Toole developed $26.6 \%(n=4)$ of the apps included within this review. Similarly, Social Skill Builder, Inc., developed 26.6\% $(n=4)$ of included apps and Shine Ireland has developed $13.3 \%(n=2)$ of apps. The remaining $33.3 \%(n=5)$ apps were developed by a variety of independent app developers (see Table 3).

Results indicate that apps with the same developer incorporated significantly more $\mathrm{BCP}$ throughout their apps and also utilized the same BCP across the apps, despite apps progressing to different levels or targeting a different skill. Apps that had the same developers incorporated between 
Table 3 Apps presented by mean quality score

\begin{tabular}{|c|c|c|c|c|c|c|c|}
\hline \multirow[t]{2}{*}{ App } & \multirow[t]{2}{*}{ App developer } & \multicolumn{4}{|c|}{ Section } & \multirow[t]{2}{*}{ Quality score } & \multirow{2}{*}{$\begin{array}{l}\text { Subjective } \\
\text { quality } \\
\text { score }\end{array}$} \\
\hline & & A & B & $\mathrm{C}$ & $\mathrm{D}$ & & \\
\hline Circles Social Skills Utility & James Stanfield Co., Inc & 4.6 & 4.75 & 5 & 4.16 & 4.63 & 3.5 \\
\hline Social Detective & Social Skills Builder, Inc & 4 & 5 & 4.33 & 5 & 4.58 & 4.5 \\
\hline Social Skills for Autism 2: Kloog 2 & Shine Ireland & 4.2 & 4.75 & 4.66 & 4.33 & 4.49 & 4 \\
\hline My School Day & Social Skills Builder, Inc & 4.4 & 5 & 4 & 4.5 & 4.48 & 4.5 \\
\hline Social Skills for Autism 3: Cosmic Classroom & Shine Ireland & 3.6 & 4.75 & 5 & 4.33 & 4.42 & 3.75 \\
\hline Conversation Skills & Janine Toole & 4.2 & 5 & 4 & 4.5 & 4.42 & 2 \\
\hline Social Detective Intermediate & Social Skills Builder, Inc & 4 & 5 & 4.33 & 4.33 & 4.42 & 4.5 \\
\hline Social Detective Advanced & Social Skills Builder, Inc & 3.8 & 5 & 4.66 & 4.16 & 4.40 & 4.5 \\
\hline Social Skills with Billy & Virtual Speech Center Inc & 3.6 & 5 & 4.6 & 4.3 & 4.38 & 4.5 \\
\hline Do it... Or not? Social Skills for ASD & Janine Toole & 4 & 5 & 4.3 & 4.16 & 4.37 & 4.25 \\
\hline Conversation Planner SE & Janine Toole & 3.8 & 5 & 4.6 & 3.6 & 4.25 & 3.75 \\
\hline Say it... Or not? Social Filter Skills, School ED & Janine Toole & 3.4 & 4.75 & 3.66 & 4 & 3.95 & 3.75 \\
\hline I Know How You Feel & Creative Social Learning, LLC & 3.2 & 5 & 3.33 & 3.5 & 3.75 & 3.25 \\
\hline Let's Be Social: Social Skills Development & Everyday Speech $L L C$ & 2.8 & 5 & 4 & 3.16 & 3.74 & 2.75 \\
\hline Peppy Pals Social Skills & Peppy Pals AB & 3 & 3.5 & 3.33 & 2.5 & 3.08 & 1.75 \\
\hline
\end{tabular}

Note: Mean Score for $\mathrm{A}=$ engagement, $\mathrm{B}=$ functionality, $\mathrm{C}=$ aesthetics, $\mathrm{D}=$ information

5 and 8 BCP across apps with a mean of 5.6 BCP in each app, in comparison to apps with independent developers that included only 3 BCP in each app. The apps developed by Janine Toole $(n=4)$ incorporated 6 different BCP, with all apps including the same $5 \mathrm{BCP}$ (token economy, discrimination training, positive reinforcement, conditioned reinforcers, and discrete trials) and one app including an additional procedure (maintenance). These procedures were presented in the same form across all apps.

Apps developed by Social Skill Builder, Inc. $(n=4)$, included 8 different BCP. All apps included the same 4 BCP (positive reinforcement, discrete trials, modelling, and discrimination training), 3 apps (75\%) incorporating the same 2 BCP (prompts and conditioned reinforcers), 1 app (25\%) incorporating rules, and 1 app (25\%) using the BCP of maintenance. It should be noted that these additional BCP existed within the first 2 apps in a series of apps (social detective, social detective intermediate) and were not included in the final app in the series.

Apps developed by Shine Ireland $(n=2)$ embedded 6 different BCP within their apps. All apps included the same 5 BCP (positive reinforcement, rules, conditioned reinforcers, discrimination training, and maintenance and generalization). The BCP of modelling was included on one app. Procedures were presented similarly across apps.

\section{Quality Assessment}

The apps included within this review have a mean quality score of 4.22 with the lowest app scoring 3.08, Peppy Pals
Social Skills, and the highest scoring app receiving a score of 4.63, Circles Social Skills Utility. Each app was rated individually to obtain a mean score for each section and an overall quality score (see Table 3 ) where apps are rank by their overall quality score.

The apps included in this review scored a mean engagement score of 3.77 (ranging from 4.6 to 2.8). Apps that obtained a high engagement score were those that were fun and interesting, customizable, allow user input and provide feedback, and were appropriate for the target audience. Apps that scored high on engagement were Circles Social Skills Utility, My School Day, Conversation Skills, and Social Skills for Autism 2: Kloog 2.

Apps that scored high on functionality were those that had fast app features, were easy to navigate/use, and were consistent and logical across screens. The apps included in this review scored a mean functionality score of 4.83 (ranging from the highest score of 5 to 3.5). A large percentage of apps scored the highest score of $5(66.6 \%$, $n=10)$ : Social Detective, My School Day, Conversation Skills, Social Skills Intermediate, Social Detective Advanced, Social Skills with Billy, Do it... Or Not? Social Skills for ASD, Conversation Planner SE, I Know How You Feel, and Let's Be Social: Social Skills Development.

The apps included in this review scored a mean aesthetics score of 4.25 (ranging from 5 to 3.33). Apps that obtained a high aesthetics score pertained to the app having good resolution, visual appeal, and appropriate arrangement of buttons/icons/content. These apps were Social Skills for Autism 2: Kloog 2, Social Detective 
Advanced, Social Skills with Billy, and Conversation Planner SE.

The apps included in this review received a mean information score of 4.03 (ranging from 5 to 2.5). Apps that scored high on this section are those that contain what they describe in the description, have specific and measurable goals, have correct and well-written content, have comprehensions but concise information, visually explain topics, and come from a legitimate source with an evidence base. These apps were Social Detective, My School Day, and Conversation Skills.

Results indicate that apps with the same developer incorporate and present the BCP similarly across apps; this is also evident in the mean quality score of apps with apps that present with the same developer scoring a similar quality score. Apps developed by Social Skill Builder, Inc., have a mean quality score of 4.47. Apps developed by Shine Ireland have a mean quality score of 4.38 and apps developed by Janine Toole have a mean quality score of 4.25 , in contrast to apps developed by independent app developers that have a lower mean score of 3.92.

Apps included in this review have a mean subjective quality score of 3.68 (ranging from 4.5 to 1.75). Apps that scored high on the subjective quality score were those that the rater would recommend to a friend, would use repeatedly over 12 months, would pay for, and were given an overall high star rating.

\section{Discussion}

The aim of the current review was to examine apps targeted at teaching social skills to individuals with ASD in order to identify the BCPs present within the app. Apps were categorized and reviewed for the $\mathrm{BCP}$ they incorporated using the TL5. Overall findings of this review indicate that the number of BCPs incorporated within apps is relatively high and the categories of the BCP were as expected for a skills teaching app. However, how the BCPs are presented and quality scores of apps appear to vary across app developers. The current review found the apps currently available via app stores using BCPs vary in the social skills they target and the standard of the BCP varies across apps and developers. A large variation was also noted across pricing and age ranges that the apps aim to target.

Apps included within this review targeted a variety of different social skills. This is promising for practitioners who have experience with behaviour change and who may seek more specific target skills to tailor teaching to individuals or clients; however, there is no evidence to support that these apps are successful in targeting these skills. As well as this, the large variety of apps that exist upon initial searches may cause confusion for a parent or caregiver who is seeking out a social skill app for their child or someone they are working with.

Of the apps included within the review, clear descriptions of the skills targeted within the apps were provided. However, a limitation of many of the included apps was that they failed to directly teach the skill. Many apps involved the use of procedures to increase skill acquisition and reinforcement-based procedures to strengthen the behaviour, but failed to directly present what the target skill is or incorporate procedures to model the skills to the user or use instructions or rules to explain the skill to the user. These are crucial elements for apps that claim to teach social skills, particularly cases where parents may be using the app as an alternative to other social skills interventions. This raises the question, if the apps do not directly teach the target skills as described should they be utilized more as a resource to support maintenance after a skill has been taught directly. For example, they could be used as an at home support for parents or caregivers after a social skills group, as opposed to relying on them solely to teach the relevant skills. This is a vital element of apps and it is important when apps are marketed that this information is made clearer for parents, caregivers, and practitioner who seek out apps. Although these apps may be beneficial for recall of the skill presented within them, this does not mean that the app user will generalize the skill which is ultimately the long-term goal.

The price and suitable age range of apps varied considerably across those included within this review. Given that some apps serve the purpose of maintaining skills, rather than skill acquisition, it could be concluded that the cost for some apps does not reflect the quality of the information and content within the app, in particular if they do not directly teach the target skills. Furthermore, many of the apps that are included within this review include levels that once they are completed, the app is no longer useful for that particular student. For example, many apps within this review consist of levels 1, 2, 3, and so on. Once these levels are completed, the app user would either need to restart the app or purchase a follow-on app if it exists. Restarting the app would not be appropriate as many social skills within the apps build onto one another and going back to the beginning may cause confusion and frustration for the app user; therefore, a follow-on app would be the best option for the student. This may not be beneficial for parents or caregivers who may be using apps instead of other forms of direct intervention, as it is not costeffective. However, this could be advantageous for teachers and practitioners who may pay for the apps and could reuse them repeatedly across clients and students.

The variety of age ranges that apps target is advantageous for sourcing an app that is suitable for particular clients or for parents who seek apps for their children. However, a large portion of the apps included within the review do not specify the age range they are suitable for and have the 
iTunes automatic age range of $4+$ which is not always accurate. This is a fundamental element that app descriptions have failed to address and is vital especially when practitioners and parents are sourcing appropriate content for individuals with ASD. Apps that have an automatic rating of $4+$ may not reach the target audience the app was designed for and may be overlooked by parents and practitioners who are likely to assume the app rating is accurate.

The categories of BCPs presented within apps to teach social skills was as expected, with apps incorporating procedures to increase skill acquisition and reinforcementbased procedures evident across all apps, showing that app developers have a knowledge of the basic principles that can be used to teach a target skills. For example, extensive research supports the use of video models to teach social skills and apps reflected this by incorporating video models and characters modelling incorrect and correct social behaviours. A further expected finding of this review was the lack of reinforcement-based procedures to weaken behaviours; this was an expected finding as skills teaching interventions usually do not aim to weaken a behaviour, instead the goal is to strengthen target behaviours. However, an unexpected finding of the current review was the lack of maintenance and generalization procedures across apps with this section being one of the lowest. This is a cause for concern due to the mode of delivery of the skills teaching; users should have the opportunity to generalize the skills learned, in particular in the natural environment. As mentioned, individuals with ASD have an affinity to technology such as iPads and tablets and research indicates that individuals with ASD prefer this method of teaching (Hourcade et al., 2013). It is vital to ensure that the skills learned are not just utilized within the app setting. Target skills should be practised and extended to the natural environment as that is where the behaviours are most likely to be reinforced and therefore maintained. Furthermore, the lack of maintenance procedures within apps is also a concern. Maintenance procedures are an important element in particular within social skills as target skills throughout the apps appear to build upon one another. For example, initiating a conversation may move onto continuing a conversation. Apps that fail to incorporate maintenance procedures in particular within an app setting which is a contrived environment may lead to users losing a previously mastered skill which may in turn affect the students' understanding and ability to perform the next target skill.

A variety of BCPs exist across apps for improving social skills for individuals with ASD; however, when an operational definition of the existing BCP is examined according to the TL5 and compared to how the BCP is presented within the app, it is clear that in some cases, the standard of these procedures is not always high. For example, there are some elements of a "gold standard" discrete trial missing such as prompts and correction procedures. The use of a correction or corrective feedback is limited within the apps with only 2 apps showing evidence of this; this is also evident with the use of prompts with only two apps incorporating prompts. The lack of these key elements across behaviour procedures causes concern for the standard of the procedures included within apps to teach social skills. These key elements are expected within apps as they are a fundamental part of procedures to increase skill acquisition that can be easy to programme and automate; without these elements, the user is not redirected to perform the correct response and fails to learn the correct response without a correction procedure. This omission could cause frustration for the user and affect the rate of skill acquisition. This inconsistency in the standard of BCP can be noted across other procedures such as positive reinforcement. Similarly, to discrete trials, positive reinforcement can only claim to be in effect when an increase in the target response is observed. However, many apps do not measure or have a baseline of the target behaviour which prohibits the ability to evaluate the effects of the app and the BCPs to determine if they are successful in increasing the skill targeted.

It should be noted that this is not the case across all procedures, procedures such as discrimination training, rules, token economies, modelling, and generalization are presented across apps and are consistent with their operational definitions. Although generalization and modelling procedures were presented accurately according to their operational definition, it is important to highlight that the content used to display modelling and promote generalization in some apps may be difficult to relate to for some users. Some apps use videos that were used in video models were quite outdated and involved scenarios such as within American high schools that may not always be relatable for users. This may in turn affect the generalization of the content presented within the apps.

\section{Limitations}

The current review is not without its limitations. Firstly, the use of the MARS as the mechanism for the quality assessment could be considered a limitation given its subjective nature. The MARS poses questions regarding how engaging the app was, if the app was interesting to use, how visually appealing the app was, did it function correctly, its visual appeal of the app etc. These questions could be answered differently depending on the raters personal opinion of the app itself; furthermore, the questions relating to how the app functions could have been skewed depending on other variables such as if the raters iPad was slow and what version iPad the rater used. This was clearly reflected in the poor IRA score for the MARS. The poor IRA score further highlights the subjective nature of the MARS and how some of the questions it poses could be open to interpretation and 
scores can vary considerably across raters. However, we believe that the quality indicator should only be used as a guide and that the most important information for users is the number of behaviour change procedures included within the app, which are most likely to contribute to skill acquisition.

A second limitation of the current study is that the apps that were selected for inclusion were not trialled by individuals with ASD to get an accurate measure of the quality of the apps and also the standard of BCPs embedded within the apps. This may have provided more clarity on certain aspects of the review such as the previous concerns about how the BCPs were presented. For example, does the positive reinforcement embedded within the apps function as a reinforcer for the target population? Monitoring an individual using the app would have increased the accuracy of the quality scores.

Future reviews should source a more objective quality assessment measure; future research may also benefit from trialling the apps with individuals with ASD to provide more information on the standard of the BCP within the apps and if the apps show an increase in the target behaviours.

\section{Considerations for App Purchasers}

The current review has acknowledged that a variety of people may be looking to purchase social skills apps, such as parents, practitioners, and teachers. Based on the information in the current review app purchasers should explore the app stores to ensure the app they choose is suitable for their purpose. App store inconsistencies in price, age range, and skills targeted can make it difficult for purchasers to do this; therefore, further research may be required prior to purchasing, for example, finding app developers websites to determine age ranges of apps or additional app reviews etc. App purchasers should also be aware that a more expensive app does not necessarily determine it as high quality, in particular for parents who may not be able to reuse the apps across different children. It is important for app purchasers to understand the context of where and when it may be suitable to use apps included in this review. Results indicate that although apps include a variety of BCPs, it could be concluded that many may not be useful as the sole intervention to target social skills for individuals with ASD. Parents who seek out social skills apps for their children should be aware that the apps included within this review may be more beneficial as an additional support to a social skills intervention rather than a replacement for direct intervention. For example, where an individual may attend a social skills group, these apps could be used at home to ensure maintenance of the skills taught. Apps included within this review may also be a useful tool within social skills groups for practitioners to support maintenance and give app users an opportunity to engage in the skill in different scenarios which may aid generalization of the skills that practitioners may be directly teaching. Prior to purchasing an app that targets social skills, parents should seek support from a clinician or practitioner who has knowledge of $\mathrm{BCP}$ procedures and the goals of the app user. App purchasers should also consider that based on the apps reviewed, it cannot be confirmed if they result in a significant increase in target behaviours. Should parents, clinicians, or other app users want to assess the level of behaviour change, they may need to use additional tools to monitor and analyze this such as collecting a baseline for target behaviours. However, this may only be feasible for practitioners with knowledge of data collection, data trends etc.

\section{How to Improve Future Apps}

There are many suggestions that can be considered for future developers of apps to teach social skill to individuals with ASD. Firstly, apps should be clear on how the skills will be targeted within the apps and how the apps should be used. It is vital for the user and those purchasing apps (parents, caregivers, teacher, practitioners etc.) to have a clear understanding of how the content is presented within the apps and if further supports will be required along with the app, for example, if a particular skill needs to be directly taught prior to app use or will the user be able to use the app alone or does it require support.

Currently, many of the apps claim to teach target skills but fail to present these within the app content. Clearly, outlining how the information will be taught will help app purchasers decide if the app is appropriate for their needs and if they have the skills to support the individual throughout the app usage. For example, if particular skills need to be taught prior to app usage, parents or caregivers may not have the skill set or knowledge to support the user. Future apps should highlight or provide additional resources for parents and users where a curriculum or videos can be used to teach the skill directly prior to app use if this information is not included within the app itself to allow for a more cost-effective app.

Secondly, the standard of BCPs within apps should be examined more closely, in particular using the TL5. Although current apps include a variety of BCPs, the standard and how they are presented is questionable. Introducing small changes such as prompts (prior to the incorrect answer) and correction procedures when an incorrect response is presented will increase skill acquisition for the user. Furthermore, generalization and maintenance procedures are fundamental procedures in future app development. Each level of the app should embed maintenance procedures of previously mastered skills to ensure the user continually is developing their skill set while practising learned target behaviours. Generalization should be 
incorporated throughout apps through different scenarios, pictures, characters etc., but users should also be encouraged to generalize the skill in the natural environment. This could be achieved by introducing "practice" rounds or homework where users have an opportunity to practise the skill in real life. To further eliminate limitations relating to how BCPs and questions surrounding if certain BCPs are functioning correctly, for example conditioned reinforcers and positive reinforcement. It may be useful for apps to take a baseline measure or provide choice in reinforcers for the user to ensure that these procedures are resulting in an increase in the target behaviour throughout the apps. Having more options and choice throughout will not only make the BCPs more effective but will also allow for reuse of the apps with different clients, making them more cost-effective.

A final recommendation for future app development is for app developers who have a number of social skills apps on the market. It may be useful for app developers to build on the apps they have and include more BCPs and vary how these BCP are presented. A large portion of the apps included within this review have the same app developer; although the apps target different social skills, the BCPs are presented the same across apps, with most of them containing the same number of BCPs. It may be beneficial for app developers to examine the apps relating to the TL5 and incorporate more evidence-based procedures to build on the quality of the apps that exist and future apps that may be developed. Developers could also work collaboratively with behaviour analysts who can provide detailed input on the most effective BCPs for skill acquisition. This could help in developing more guidelines for app developers on the procedures that can be used for skills teaching, maintenance, and generalization or creating a standard for apps stating examples of evidence-based procedures that must be included within apps before they can be marketed as apps that teach social skills. This will aid both app developers as well as practitioners and parents who are seeking apps.

Currently, the apps that exist to teach social skills to individuals with ASD incorporate a number of evidencebased procedures according to the TL5 that are successful in increasing skill acquisition for individuals with ASD. Although the existing apps incorporate BCPs that would be expected from a skill teaching app, the apps vary in price, the skills they target, the number of BCPs present within the apps, and the quality score of the apps. The current review provides recommendations for future app development to ensure social skills apps incorporate evidence-based procedures to ensure high-quality and consistency within-app development.
Funding Open Access funding provided by the IReL Consortium

\section{Declarations}

Conflict of Interest The authors declare no competing interests.

Open Access This article is licensed under a Creative Commons Attribution 4.0 International License, which permits use, sharing, adaptation, distribution and reproduction in any medium or format, as long as you give appropriate credit to the original author(s) and the source, provide a link to the Creative Commons licence, and indicate if changes were made. The images or other third party material in this article are included in the article's Creative Commons licence, unless indicated otherwise in a credit line to the material. If material is not included in the article's Creative Commons licence and your intended use is not permitted by statutory regulation or exceeds the permitted use, you will need to obtain permission directly from the copyright holder. To view a copy of this licence, visit http://creativecommons.org/licenses/by/4.0/.

\section{References}

American Psychiatric Association. (2013). DSM-5 diagnostic classification. Diagnostic and Statistical Manual of Mental Disorders. https://doi.org/10.1176/appi.books.9780890425596.x00diagnos ticclassification

Behavior Analyst Certification Board. (2017). BCBA/BCaBA task list (5th ed.). Author. https://www.bacb.com/ bcba-bcaba-task-list-5th-ed/.

Boyd, B. A., Conroy, M. A., Asmus, J. M., McKenney, E. L. W., \& Mancil, G. R. (2008). Descriptive analysis of classroom setting events on the social behaviors of children with autism spectrum disorder. Education and Training in Developmental Disabilities. http://www.jstor.org/stable/23879929.

Camargo, S. P. H., Rispoli, M., Ganz, J., Hong, E. R., Davis, H., \& Mason, R. (2014). A review of the quality of behaviorally-based intervention research to improve social interaction skills of children with ASD in inclusive settings. Journal of Autism and Developmental Disorders. https://doi.org/10.1007/s10803-014-2060-7

Conroy, D. E., Yang, C. H., \& Maher, J. P. (2014). Behavior change techniques in top-ranked mobile apps for physical activity. American Journal of Preventive Medicine. https://doi.org/10.1016/j. amepre.2014.01.010

Crooke, P. J., Hendrix, R. E., \& Rachman, J. Y. (2008). Brief report: Measuring the effectiveness of teaching social thinking to children with Asperger syndrome (AS) and high functioning autism (HFA). Journal of Autism and Developmental Disorders. https://doi.org/ 10.1007/s10803-007-0466-1

Dillenburger, K., Keenan, M., Gallagher, S., \& McElhinney, M. (2004). Parent education and home-based behaviour analytic intervention: An examination of parents' perceptions of outcome. Journal of Intellectual and Developmental Disability. https://doi.org/10. 1080/13668250410001709476

Draper Rodríguez, C., Strnadová, I., \& Cumming, T. (2014). Using iPads with students with disabilities: Lessons learned from students, teachers, and parents. Intervention in School and Clinic. https://doi.org/10.1177/1053451213509488

Flynn, L., \& Healy, O. (2012). A review of treatments for deficits in social skills and self-help skills in autism spectrum disorder. Research in Autism Spectrum Disorders. https://doi.org/10.1016/j. rasd.2011.06.016

Grossard, C., Grynspan, O., Serret, S., Jouen, A. L., Bailly, K., \& Cohen, D. (2017). Serious games to teach social interactions and 
emotions to individuals with autism spectrum disorders (ASD). Computers and Education. https://doi.org/10.1016/j.compedu. 2017.05.002

Hourcade, J. P., Williams, S. R., Miller, E. A., Huebner, K. E., \& Liang, L. J. (2013). Evaluation of tablet apps to encourage social interaction in children with autism spectrum disorders. Conference on Human Factors in Computing Systems - Proceedings. https://doi. org/10.1145/2470654.2466438

Johnston, J. M., Mellichamp, F. H., Shook, G. L., \& Carr, J. E. (2014). Determining BACB examination content and standards. Behavior Analysis in Practice, 7(1), 3-9. https://doi.org/10.1007/ s40617-014-0003-6

Kim, J. W., Nguyen, T.-Q., Gipson, S.Y.-M.T., Shin, A. L., \& Torous, J. (2018). Smartphone apps for autism spectrum disorder-Understanding the evidence. Journal of Technology in Behavioral Science. https://doi.org/10.1007/s41347-017-0040-4

Mason, R. A., Rispoli, M., Ganz, J. B., Boles, M. B., \& Orr, K. (2012). Effects of video modeling on communicative social skills of college students with Asperger syndrome. Developmental Neurorehabilitation. https://doi.org/10.3109/17518423.2012.704530

McCoy, A., Holloway, J., Healy, O., Rispoli, M., \& Neely, L. (2016). A systematic review and evaluation of video modeling, role-play and computer-based instruction as social skills interventions for children and adolescents with high-functioning autism. Review Journal of Autism and Developmental Disorders. https://doi.org/ 10.1007/s40489-015-0065-6

Mesibov, G. B., \& Shea, V. (2011). Evidence-based practices and autism. Autism. https://doi.org/10.1177/1362361309348070

Mintz, J., Branch, C., March, C., \& Lerman, S. (2012). Key factors mediating the use of a mobile technology tool designed to develop social and life skills in children with autistic spectrum disorders. Computers and Education. https://doi.org/10.1016/j.compedu. 2011.07.013

Morrissey, E. C., Corbett, T. K., Walsh, J. C., \& Molloy, G. J. (2016). Behavior change techniques in apps for medication adherence: A content analysis. American Journal of Preventive Medicine. https://doi.org/10.1016/j.amepre.2015.09.034.

Odom, S. L., Thompson, J. L., Hedges, S., Boyd, B. A., Dykstra, J. R., Duda, M. A., Szidon, K. L., Smith, L. E., \& Bord, A. (2015). Technology-aided interventions and instruction for adolescents with autism spectrum disorder. Journal of Autism and Developmental Disorders. https://doi.org/10.1007/s10803-014-2320-6

Papadakis, S., \& Kalogiannakis, M. (2017). Mobile educational applications for children: What educators and parents need to know. International Journal of Mobile Learning and Organisation. https://doi.org/10.1504/IJMLO.2017.085338

Ploog, B. O., Scharf, A., Nelson, D., \& Brooks, P. J. (2013). Use of computer-assisted technologies (CAT) to enhance social, communicative, and language development in children with autism spectrum disorders. Journal of Autism and Developmental Disorders. https://doi.org/10.1007/s10803-012-1571-3

Ramdoss, S., MacHalicek, W., Rispoli, M., Mulloy, A., Lang, R., \& O'Reilly, M. (2012). Computer-based interventions to improve social and emotional skills in individuals with autism spectrum disorders: A systematic review. Developmental Neurorehabilitation. https://doi.org/10.3109/17518423.2011.651655

Reed, F. D., Hyman, S. R., \& Hirst, J. M. (2011). Applications of technology to teach social skills to children with autism. Research in Autism Spectrum Disorders. https://doi.org/10.1016/j.rasd.2011. 01.022

Reichow, B. (2011). Development, procedures, and application of the evaluative method for determining evidence-based practices in autism. In Evidence-Based Practices and Treatments for Children with Autism.https://doi.org/10.1007/978-1-4419-6975-0_2.

Reichow, B., \& Volkmar, F. R. (2010). Social skills interventions for individuals with autism: Evaluation for evidence-based practices within a best evidence synthesis framework. Journal of Autism and Developmental Disorders. https://doi.org/10.1007/ s10803-009-0842-0

Scott, C., De Barra, M., Johnston, M., De Bruin, M., Scott, N., Matheson, C., Bond, C., \& Watson, M. C. (2020). Using the behaviour change technique taxonomy v1 (BCTTv1) to identify the active ingredients of pharmacist interventions to improve non-hospitalised patient health outcomes. British Medical Journal Open. https://doi.org/10.1136/bmjopen-2019-036500

Shic, F., \& Goodwin, M. (2015). Introduction to technologies in the daily lives of individuals with autism. Journal of Autism and Developmental Disorders. https://doi.org/10.1007/ s10803-015-2640-1

Stoyanov, S. R., Hides, L., Kavanagh, D. J., Zelenko, O., Tjondronegoro, D., \& Mani, M. (2015). Mobile app rating scale: A new tool for assessing the quality of health mobile apps. JMIR MHealth and UHealth. https://doi.org/10.2196/mhealth.3422

Van Houten, R., Axelrod, S., Bailey, J. S., Favell, J. E., Foxx, R. M., Iwata, B. A., \& Lovaas, O. I. (1988). The right to effective behavioral treatment. Journal of Applied Behavior Analysis. https://doi. org/10.1901/jaba.1988.21-381

Wainer, A. L., \& Ingersoll, B. R. (2011). The use of innovative computer technology for teaching social communication to individuals with autism spectrum disorders. Research in Autism Spectrum Disorders. https://doi.org/10.1016/j.rasd.2010.08.002

Walton, K. M., \& Ingersoll, B. R. (2013). Improving social skills in adolescents and adults with autism and severe to profound intellectual disability: A review of the literature. Journal of Autism and Developmental Disorders. https://doi.org/10.1007/ s10803-012-1601-1

Williams White, S., Keonig, K., \& Scahill, L. (2007). Social skills development in children with autism spectrum disorders: A review of the intervention research. Journal of Autism and Developmental Disorders. https://doi.org/10.1007/s10803-006-0320-x

Publisher's Note Springer Nature remains neutral with regard to jurisdictional claims in published maps and institutional affiliations. 